\title{
Stability Analysis of Stochastic Prioritized Dynamic Scheduling for Resource-Aware Heterogeneous Multi-Loop Control Systems
}

\author{
Mohammad H. Mamduhi and Adam Molin and Sandra Hirche
}

\begin{abstract}
The aim of this paper is to develop a networked resource-aware control scheme by assigning dynamic priorities to acquire a scarce resource. The networked control system is assumed to consist of a finite number of heterogeneous stochastic control systems whose feedback loops are closed over a shared constrained communication channel. The scheduler decides which subsystem utilizes the channel, and subsequently which controller is updated with real state values. We introduce a prioritized error-based (PEB) scheduling protocol which allocates the resource according to a network-induced error function, such that the likelihood to allocate the resource to a subsystem increases with the growing error norm. By using drift criteria, we prove the described system is stochastically stable under very mild conditions. Numerical simulations show that the stability of the overall NCS along with a significant control performance improvement in comparison to the other protocols.
\end{abstract}

\section{INTRODUCTION}

The consideration of networked control systems (NCS) with communication constraints has received a lot of attention in recent years. An NCS is the integration of a multitude of small-scale entities coupled through a resourceconstrained communication network. Therein, the design of the scheduling protocol for NCS has undergone several paradigm shifts in order to meet the real-time requirements of the control tasks. One of the basic paradigm changes is introduced in [1] by the try-once-discard (TOD) protocol. The TOD protocol uses only current measurement data for transmission and discards the blocked data. The protocol dynamically prioritizes transmissions by choosing the measurement with the largest discrepancy between its actual value and its estimate at the controller. A central role in the stability analysis of systems with deterministic communication plays the maximal allowable transfer interval (MATI) denoting the upper bound on the interval of two successive transmissions to guarantee stability of the NCS [1]-[3]. In wireless communication systems, contentionbased protocols, like CSMA, are intrinsically stochastic. These protocols that are the subject of this work however do not allow to use the notion of MATI in general, as the intervals between transmissions usually can not be bounded uniformly with probability one. An extension to stochastic protocols and communication systems with random packet

M. H. Mamduhi, A. Molin and S. Hirche are with the Institute for Information-oriented Control, Technische Universität München, Arcisstraße 21, D-80290 München, Germany; http: / / www.itr.ei.tum.de, mh. mamduhi@tum. de,adam. molinetum. de,

hirchedtum.de. Research supported by the German Research Foundation (DFG) within the Priority Program SPP 1305 "Control Theory of Digitally Networked Dynamical Systems“. dropouts is given in [4]-[6]. Stability conditions for mean square stability and $L_{p}$ stability-in-expectation are derived via Lyapunov theory.

With regard to the existing literature, the novelty of this work is given by considering a stochastic protocol over a resource-constrained communication channel whose priorities are error-dependent. As the errors are driven by the stochastic noise process, transmissions occur randomly in an event-based fashion. The approach uses the scarce resource of communicating more efficiently to stabilize the system and decreases the mean variance of the error in comparison with static protocols, such as round robin and CSMA. Moreover, the probabilistic nature of the protocol facilitates an approximative decentralized implementation through errordependent back-off exponents. The system under consideration requires novel methods to analyze the asymptotic behavior of the NCS. Our contribution is to analyze the stability properties of a networked system consisting of multiple loops closed over a shared communication network by employing a stochastic scheduler with dynamically assigned priorities. We assume that we are given stabilizing state-feedback controllers in case of ideal communication. If no state update is received, a model-based state observer implemented at the controller predicts the state evolution. Inspired by the idea of error-dependent intensity for transmission, [7], we introduce a stochastic protocol denoted as prioritized errorbased (PEB) protocol. The PEB protocol assigns to each subsystem a probability of utilizing the resource according to the individual networked-induced error. As stochastic disturbances are considered opposed to [4], [5], we relax the notion of stochastic stability to ergodicity with finite second moment of the Markov chain. Using drift criteria [8], we show that the overall system is ergodic. The key idea in stability analysis is to consider multiple time steps in order to show that the drift of the Lyapunov function is negative. Straight-forward arguments indicate that the stability results can accommodate event-triggered scheduling schemes within the prioritizing protocol to further reduce communication traffic [9]-[13]. An extension of this work so called $p$ powered PEB, which uses more conservative bounds on the drift, is also discussed in [14]. Numerical results illustrate the stability properties of the proposed scheme. They indicate an increased performance in terms of the mean squared networked-induced estimation error compared to the round robin and CSMA schemes.

The remainder of this paper is structured as follows. Problem statement is described in Section II. Section III starts with some preliminaries of stochastic stability and 
ergodicity, and then proceeds with the stability analyses. The efficiency of the proposed approach is illustrated in Section IV by numerical simulations.

Notation. In this paper, the Euclidean norm is denoted by $\|\cdot\|_{2}$. The expectation and conditional expectation operators are denoted by $\mathrm{E}[\cdot]$ and $\mathrm{E}[\cdot \mid \cdot]$. The relation $\mathcal{N}(0, X)$ denotes a zero-mean Gaussian random variable with covariance matrix $X$. If not otherwise stated, a state variable with superscript $i$ indicates that it belongs to subsystem $i$. For constant matrices though, subscript $i$ indicates the corresponding subsystem and superscript $n$ denotes the matrix power.

\section{PROBLEM STATEMENT}

We consider a networked system composed of $N$ independent heterogeneous subsystems which are coupled through a shared communication channel. Each individual control subsystem consists of a linear time invariant stochastic plant $\mathcal{P}^{i}$, a stabilizing state-feedback controller $\mathcal{C}^{i}$, and a sensor $\mathcal{S}^{i}$. An event-based global scheduler situated at the communication channel receives the data, in form of error norms, from all sensors and decides if the state is an event to be scheduled for channel utilization. This is illustrated graphically in Fig. 1. The process $\mathcal{P}^{i}$ is modeled by a stochastic process evolving by the following difference equation

$$
x_{k+1}^{i}=A_{i} x_{k}^{i}+B_{i} u_{k}^{i}+w_{k}^{i},
$$

where $A_{i} \in \mathbb{R}^{n_{i} \times n_{i}}, B_{i} \in \mathbb{R}^{n_{i} \times m_{i}}$. The state vector $x_{k}^{i}$ and control input $u_{k}^{i}$ are taking values in $\mathbb{R}^{n_{i}}$ and $\mathbb{R}^{m_{i}}$, respectively. The system noise $w_{k}^{i} \in \mathbb{R}^{n_{i}}$ is i.i.d. with $w_{k}^{i} \sim$ $\mathcal{N}\left(0, W_{i}\right)$ at each time-step $k$. For notational convenience, we assume that the system noise is unity variance Gaussian distribution, but the results hold for arbitrary positive definite $W_{i}$. Since, the stability analysis is independent of initial states, $x_{0}^{i}, i \in\{1, \ldots, N\}$, might have any arbitrary distribution with bounded second moment. The scheduler output at time-step $k$ is described by $\delta_{k}^{i} \in\{0,1\}$ as follows

$$
\delta_{k}^{i}= \begin{cases}1 & \text { subsystem } i \text { is updated } \\ 0 & \text { subsystem } i \text { is blocked }\end{cases}
$$

and implies the received signal $z_{k}$ at the controller as

$$
z_{k}^{i}= \begin{cases}x_{k}^{i} & \delta_{k}^{i}=1 \\ \varnothing & \delta_{k}^{i}=0\end{cases}
$$

It is assumed that sensor and controller of the $i$ th subsystem merely have knowledge of $A_{i}, B_{i}, W_{i}$, the distribution of $x_{0}$. Then control law $\gamma^{i}$ is described by causal mappings of the past observations for each time step $k$, i.e.

$$
u_{k}^{i}=\gamma_{k}^{i}\left(Z_{k}^{i}\right)=-L_{i} \mathrm{E}\left[x_{k}^{i} \mid Z_{k}^{i}\right]
$$

where $Z_{k}^{i}=\left\{z_{0}^{i}, \ldots, z_{k}^{i}\right\}$ is the $i$ th subsystem observation history, and the $L_{i}$ is the stabilizing feedback gain. We assume that each loop is stabilized with the state feedback controller in (2) in case of ideal communication. The controllers are updated by an estimator, in case of a blocked data transmission request, only if the closed-loop matrix $\left(A_{i}-B_{i} L_{i}\right)$ is Hurwitz, i.e. if $\delta_{k}^{i}=0$, then

$$
\mathrm{E}\left[x_{k}^{i} \mid Z_{k}^{i}\right]=\left(A_{i}-B_{i} L_{i}\right) \mathrm{E}\left[x_{k-1}^{i} \mid Z_{k-1}^{i}\right]
$$

with the initial condition $\mathrm{E}\left[x_{0}^{i} \mid Z_{0}^{i}\right]=0$. For each subsystem $i$, the network-induced error state $e_{k}^{i} \in \mathbb{R}^{n_{i}}$ is defined as $e_{k}^{i}=x_{k}^{i}-\mathrm{E}\left[x_{k}^{i} \mid Z_{k-1}^{i}\right]$ which evolves as follows

$$
e_{k+1}^{i}=\left(1-\delta_{k}^{i}\right) A_{i} e_{k}^{i}+w_{k}^{i}
$$

The augmented state $\left[x_{k}^{i}, e_{k}^{i}\right]$ has a triangular dynamics within each subsystem, according to (1)-(4)

$$
\left[\begin{array}{c}
x_{k+1}^{i} \\
e_{k+1}^{i}
\end{array}\right]=\left[\begin{array}{cc}
A_{i}-B_{i} L_{i} & \left(1-\delta_{k}^{i}\right) B_{i} L_{i} \\
0 & \left(1-\delta_{k}^{i}\right) A_{i}
\end{array}\right]\left[\begin{array}{c}
x_{k}^{i} \\
e_{k}^{i}
\end{array}\right]+\left[\begin{array}{c}
w_{k}^{i} \\
w_{k}^{i}
\end{array}\right]
$$

which underlines the system state $x_{k}^{i}$ does not affect evolution of $e_{k}^{i}$. Thus, showing the sequence $e_{k}$ is stable implies the overall system's stability. The prioritized error-based (PEB) scheduling policy defines the probability of channel access for each subsystem at time $k$ as

$$
\mathrm{P}\left[\delta_{k}^{i}=1 \mid e_{k}^{j}, j \in\{1, \ldots, N\}\right]=\frac{\left\|e_{k}^{i}\right\|_{2}^{2}}{\sum_{j=1}^{N}\left\|e_{k}^{j}\right\|_{2}^{2}}
$$

According to (5), the highest error has the channel access priority, and other requests accompanied with lower priorities are likely dropped. As the scheduling policy is memoryless, the process is repeated in every time step $k$. As the network allows only one transmission per time step, we have the following hard constraint with probability 1 for every $k \geq 0$

$$
\sum_{i=1}^{N} \delta_{k}^{i}=1
$$

It is straightforward to extend the approach to a different number than one of allowed transmission per time step.

Remark 1: Stochastic nature of the algorithm, enables us to implement the PEB policy approximately in a decentralized fashion. It is envisioned that every subsystem randomly determines its priority according to a probability distribution depending on its own error. In a wireless CSMA communication systems, the priority could be reflected in the error-dependent distribution of the backoff-time of each subsystem during one time step. This implies that the mean back-off time of a subsystem decreases with an increase of the error norm.

The aggregate error state $e_{k} \in \mathbb{R}^{n}$ defined as

$$
e_{k}=\left[e_{k}^{1}, \ldots, e_{k}^{N}\right]^{\top},
$$

where $n=n_{1}+\cdots+n_{N}$, is a time-homogeneous Markov chain, because the scheduling policy defined in (5) is a randomized Markov policy depending on the values of $e_{k}$.

\section{STABILITY ANALYSIS UNDER PEB SCHEDULING POLICY}

In this section, the stability of networked control systems with multiple control loops sharing a constrained communication channel, which is scheduled by the PEB scheduling 


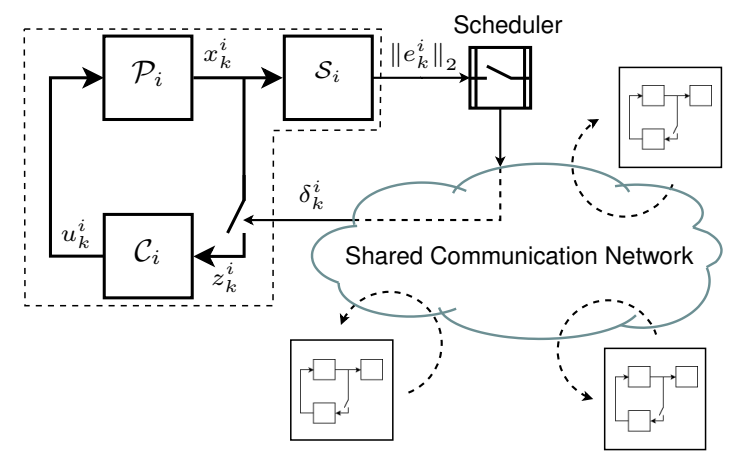

Fig. 1. A NCS with a shared communication channel

policy, is presented. We start with a networked system composed of two heterogeneous subsystems, and then extend the results for arbitrary number of subsystems. Beforehand, some preliminaries are introduced to facilitate the discussions.

\section{A. Preliminaries}

As the Markov chain defined in (7) evolves in $\mathbb{R}^{n}$, we have a Markov chain in an uncountable state space, which comprehensively discussed in [8], Chapter 14. The notions used are similar of that for countable Markov chain, however, several generalizations must be made, for example $\psi$-irreducibility, where $\psi$ is a non-trivial measure of the uncountable state space. Another important generalization is that of so-called small sets, which can be identified by compact sets in our problem. These sets take the role of finite sets in countable Markov chains. The stability concept used in this paper is given by $f$-ergodicity defined in the following.

Definition 1: Let $f \geq 1$ be a real-valued function in $\mathbb{R}^{n}$. A Markov chain $e_{k}$ is said to be $f$-ergodic, if

1) $e_{k}$ is positive Harris recurrent with invariant probability measure $\pi$

2) $\mathrm{E}[\pi(f)]$ is finite, where $\pi(f)=\int f(e) \pi(d e)$

3) for every initial value $e_{0}, \lim _{k \rightarrow \infty}\left\|P^{k}\left(e_{0}, \cdot\right)-\pi\right\|_{f}=$ 0 , where $P$ is the probability transition matrix

The $f$-norm $\|\cdot\|_{f}$ for any signed measure $\nu$ is defined as

$$
\|\nu\|_{f}=\sup _{|g| \leq f}|\nu(g)|
$$

Remark 2: A Markov chain is said to be positive Harris recurrent, if it is Harris recurrent and there exists an invariant probability measure, see section 10.1 in [8].

Remark 3: From now on, $f$ is assumed to be quadratic. Therefore, $f$-ergodicity implies that the stationary distribution of the Markov chain has a finite second moment.

The next definition gives us a notion for the gradient of the Markov chain with respect to a real-valued function of states.

Definition 2 (Drift for Markov chains, [8]): Let $V$ be a real-valued function in $\mathbb{R}^{n}$. The drift operator $\Delta$ is defined for any non-negative measurable function $V$ as

$$
\Delta V\left(e_{k}\right)=\mathrm{E}\left[V\left(e_{k+1}\right) \mid e_{k}\right]-V\left(e_{k}\right), \quad e_{k} \in \mathbb{R}^{n} .
$$

The main theorem is summarized in the following theorem which can be found on p.334 in [8].
Theorem 1 (Aperiodic Ergodic Theorem): Suppose that the Markov chain $e_{k}$ is $\psi$-irreducible and aperiodic and let $f(e) \geq 1$ be a real-valued function in $\mathbb{R}^{n}$. If a small set $\mathcal{D}$ and a non-negative real-valued function $V$ exist such that

$$
\Delta V(e) \leq-f(e), \quad e \in \mathbb{R}^{n} \backslash \mathcal{D}
$$

and $\Delta V<\infty$ for $e \in \mathcal{D}$, then the Markov chain $e_{k}$ is $f$-ergodic.

Remark 4: Since we assume Gaussian additive noise with $W_{i}>0$, the transition kernel $P(e, \cdot)$ at any state $e$ of the Markov chain $e_{k}$ has a positive density function. Then, the Markov chain is $\psi$-irreducible and aperiodic. Analogously as in the section 5.3.5 of [8], it can be concluded that all the compact sets are small.

\section{B. Two-Subsystem Network}

Consider two LTI control systems coupled through the communication network, with the plants and controllers given as (1) and (2). At each time $k$, the scheduler is provided with the error norms, and likely selects the subsystem with the highest error to access the channel. In case of dismissed transmission request, an observer predicts the state evolution. The stability analysis is performed based on the drift conditions. We first state the following crucial lemma.

Lemma 1 (Expected value of the ratio $\frac{a}{b}$ ): Suppose $\quad a$ and $b \neq 0$ are two dependent random variables, then

$$
\begin{gathered}
\mathrm{E}\left[\left.\frac{a}{b}\right|_{b \neq 0}\right]=\mathrm{E}[a] \mathrm{E}\left[\frac{1}{b}\right]+\sum_{i=1}^{\infty}(-1)^{i} \frac{\mathrm{E}\left[(a-\mathrm{E}[a])(b-\mathrm{E}[b])^{i}\right]}{\mathrm{E}[b]^{i+1}} \\
\text { Proof: See [15]. }
\end{gathered}
$$

The following theorem incurs the stability of the twosubsystem NCS employing PEB allocating strategy.

Theorem 2: Consider a NCS consists of two stochastic LTI control loops coupled through a communication channel constrained by (6), and with the stabilizing controllers $\gamma^{i}$ as defined in (2). Then, if the channel access is scheduled as introduced in (5), then the Markov chain in (7) is $f$-ergodic and has finite second moment.

Proof: According to Remark 4, the Markov chain $e_{k}$ is $\psi$-irreducible and aperiodic. Then, as in Def. 2, we introduce the non-negative measurable function $V$ as

$$
V\left(e_{k}\right)=\sum_{i=1,2}\left\|e_{k}^{i}\right\|_{2}^{2}
$$

In the following, as is also discussed in [16], we evaluate the stochastic stability over two consecutive time-steps, in terms of $f$-ergodicity and finite second moment. The Markov chain $e_{k}$ is then $f$-ergodic if

$$
\Delta^{2} V\left(e_{k}\right)=\mathrm{E}\left[V\left(e_{k+2}\right) \mid e_{k}\right]-V\left(e_{k}\right) \leq-f\left(e_{k}\right)
$$

Adjusting (4) for two consecutive time steps gives

$$
e_{k+2}^{i}=\left(1-\delta_{k+1}^{i}\right)\left(1-\delta_{k}^{i}\right) A_{i}^{2} e_{k}^{i}+\left(1-\delta_{k+1}^{i}\right) A_{i} w_{k}^{i}+w_{k+1}^{i}
$$

According to (10), $V\left(e_{k+2}\right)=\sum_{i=1,2}\left\|e_{k+2}^{i}\right\|_{2}^{2}$, therefore the statistical independence of $e_{k}$, and zero-mean variables 
$w_{k}$, and $w_{k+1}$, the second-order implies

$$
\begin{aligned}
& \Delta^{2} V\left(e_{k}\right) \\
& \quad=\sum_{i=1,2} \mathrm{E}\left[\left\|\left(1-\delta_{k+1}^{i}\right)\left(1-\delta_{k}^{i}\right) A_{i}^{2} e_{k}^{i}\right\|_{2}^{2} \mid e_{k}\right] \\
& +\sum_{i=1,2} \mathrm{E}\left[\left\|\left(1-\delta_{k+1}^{i}\right) A_{i} w_{k}^{i}\right\|_{2}^{2} \mid e_{k}\right] \\
& +\sum_{i=1,2} \mathrm{E}\left[\left\|w_{k+1}^{i}\right\|_{2}^{2}\right]-\sum_{i=1,2}\left\|e_{k}^{i}\right\|_{2}^{2}
\end{aligned}
$$

At time $k$, one subsystem transmits, suppose the 1st, i.e. $\delta_{k}^{1}=1$, and inevitably, $\delta_{k}^{2}=0$. Thus, the drift is simplified as

$$
\begin{aligned}
& \Delta^{2} V\left(e_{k}\right) \\
& \quad=\mathrm{E}\left[\left\|\left(1-\delta_{k+1}^{2}\right) A_{2} e_{k+1}^{2}\right\|_{2}^{2} \mid e_{k}\right]+\xi^{+}-\sum_{i=1,2}\left\|e_{k}^{i}\right\|_{2}^{2}
\end{aligned}
$$

where, $\xi^{+}=\mathrm{E}\left[\left\|\left(1-\delta_{k+1}^{1}\right) A_{1} w_{k}^{1}\right\|_{2}^{2} \mid e_{k}\right]+\sum_{i=1,2} n_{i}$. Here we investigate two complementary cases by introducing a binary random variable $d \in\{1,2\}$ as follows

$$
d= \begin{cases}1 & \left\|e_{k+1}^{2}\right\|_{2}^{2} \leq \varepsilon_{2}<M_{2} \\ 2 & \left\|e_{k+1}^{2}\right\|_{2}^{2}>\varepsilon_{2}\end{cases}
$$

where, $\varepsilon_{2}>0$, and $d$ occurs with probabilities $\mathrm{P}\left[d=1 \mid e_{k}\right]=\alpha_{2}$ and $\mathbf{P}\left[d=2 \mid e_{k}\right]=1-\alpha_{2}$, and $\alpha_{2} \in[0,1]$. $M_{2}$ is the maximum allowable error threshold for individual subsystem $i=2$. Employing the law of iterated expectation

$$
\begin{aligned}
\mathrm{E} & {\left[\left\|\left(1-\delta_{k+1}^{2}\right) A_{2} e_{k+1}^{2}\right\|_{2}^{2} \mid e_{k}\right] } \\
& =\mathrm{E}\left[\mathrm{E}\left[\left\|\left(1-\delta_{k+1}^{2}\right) A_{2} e_{k+1}^{2}\right\|_{2}^{2} \mid e_{k}, d\right] \mid e_{k}\right] \\
& =P\left(d=1 \mid e_{k}\right) . \mathrm{E}\left[\left\|\left(1-\delta_{k+1}^{2}\right) A_{2} e_{k+1}^{2}\right\|_{2}^{2} \mid e_{k}, d=1\right] \\
& +P\left(d=2 \mid e_{k}\right) \cdot \mathrm{E}\left[\left\|\left(1-\delta_{k+1}^{2}\right) A_{2} e_{k+1}^{2}\right\|_{2}^{2} \mid e_{k}, d=2\right] \\
& \leq \alpha_{2} \varepsilon_{2}\left\|A_{2}\right\|_{2}^{2}+\left(1-\alpha_{2}\right) \mathrm{E}_{2}\left[\left(1-\delta_{k+1}^{2}\right)\left\|A_{2} e_{k+1}^{2}\right\|_{2}^{2} \mid e_{k}\right]
\end{aligned}
$$

where we denote $\mathrm{E}\left[\cdot \mid e_{k}, d=2\right]=\mathrm{E}_{2}\left[\cdot \mid e_{k}\right]$ for abbreviation. Based on the definition of the PEB scheduling in (5),

$$
\begin{gathered}
\mathrm{E}_{2}\left[1-\delta_{k+1}^{2} \mid e_{k}\right]=\mathrm{E}_{2}\left[\frac{\left\|w_{k}^{1}\right\|_{2}^{2}}{\left\|w_{k}^{1}\right\|_{2}^{2}+\left\|w_{k}^{2}+A_{2} e_{k}^{2}\right\|_{2}^{2}} \mid e_{k}\right] \\
\leq \mathrm{E}_{2}\left[\frac{1}{\left\|w_{k}^{2}+A_{2} e_{k}^{2}\right\|_{2}^{2}} \mid e_{k}\right]=\mathrm{E}_{2}\left[\frac{1}{\left\|e_{k+1}^{2}\right\|_{2}^{2}} \mid e_{k}\right]
\end{gathered}
$$

Since, $\mathrm{P}\left[\left\|e_{k+1}^{2}\right\|_{2}^{2}>\varepsilon_{2} \mid d=2\right]=1$, we derive the upper bound for the latter based on the results in [17] as

$$
\mathrm{E}_{2}\left[\frac{1}{\left\|w_{k}^{2}+A_{2} e_{k}^{2}\right\|_{2}^{2}} \mid e_{k}\right] \leq \varepsilon_{2}^{-1}\left(1-\gamma^{-1}\left(\mu-\varepsilon_{2}\right)^{2}\right)
$$

where, $\gamma=\sigma^{2}+\mu\left(\mu-\varepsilon_{2}\right), \mu=\mathrm{E}_{2}\left[\left\|w_{k}^{2}+A_{2} e_{k}^{2}\right\|_{2}^{2} \mid e_{k}\right]=$ $n_{2}+\left\|A_{2} e_{k}^{2}\right\|_{2}^{2}$ and $\sigma^{2}=\operatorname{var}\left[\left\|w_{k}^{2}+A_{2} e_{k}^{2}\right\|_{2}^{2} \mid e_{k}, d=2\right]$. In fact, $\mu$ and $\sigma^{2}$ are taken to be approximations of the real mean and variance as they neglect that $\left\|e_{k+1}^{2}\right\|_{2}^{2}>\varepsilon_{2}$. But as $\varepsilon_{2}$ can be chosen arbitrarily small, such that it excludes close-to-zero error values and enables us to exploit (13), the deviation can be made arbitrarily small due to the absolute continuity of the distribution. In other words, $\varepsilon_{2}$ is defined just to avoid the bound in (13) becomes meaningless by close-to-zero error values. Moreover, we will be majorly concerned with the limit $\left\|A_{2} e_{k}^{2}\right\|_{2}^{2} \rightarrow \infty$, where both mean and variance also tend to infinity and approximation errors become negligible. Since, $A_{2} e_{k}^{2}$ is given, and $w_{k}^{2} \sim \mathcal{N}(0,1)$, the random variable $w_{k}^{2}+A_{2} e_{k}^{2}$ is also normally distributed according to $\mathcal{N}\left(A_{2} e_{k}^{2}, 1\right)$, and therefore, $\left\|w_{k}^{2}+A_{2} e_{k}^{2}\right\|_{2}^{2}$ is distributed as noncentral chi-squared, with $n_{2}$ degrees of freedom and noncentrality parameter $\lambda=n_{2}\left\|A_{2} e_{k}^{2}\right\|_{2}^{2}$. Thus, $\sigma^{2}=2\left(n_{2}+2 \lambda\right)=2\left(n_{2}+2 n_{2}\left\|A_{2} e_{k}^{2}\right\|_{2}^{2}\right)$. Simplifying (13), and employing Lemma 1 yield the upper bound as

$$
\begin{aligned}
\mathrm{E}_{2} & {\left[1-\delta_{k+1}^{2} \mid e_{k}\right] } \\
\leq & \frac{n_{1}}{\varepsilon_{2}}\left(1-\frac{\left(\mu-\varepsilon_{2}\right)^{2}}{2\left(n_{2}+2 n_{2}\left\|A_{2} e_{k}^{2}\right\|_{2}^{2}\right)+\mu^{2}-\mu \varepsilon_{2}}\right) \\
& -\sum_{i_{\text {(Odd })}=1}^{\infty} \frac{(i+1) ! n_{1}^{\left(\frac{i+1}{2}\right)}}{\left(\frac{i+1}{2}\right) !\left(n_{1}+n_{2}+\left\|A_{2} e_{k}^{2}\right\|_{2}^{2}\right)^{i+1}}=c_{2}
\end{aligned}
$$

It is easy to show that the value in the above parenthesis is always positive, since $\mu>\varepsilon_{2}$, and it tends to zero as $\mu$ tends to infinity. Superposing both obtained upper bounds, provides the aggregate bound on $\mathrm{E}\left[V\left(e_{k+2}\right) \mid e_{k}\right]$

$$
\begin{aligned}
\mathrm{E} & {\left[V\left(e_{k+2}\right) \mid e_{k}\right] } \\
& =\mathrm{E}\left[\left\|\left(1-\delta_{k+1}^{2}\right) A_{2} e_{k+1}^{2}\right\|_{2}^{2} \mid e_{k}\right]+\xi^{+} \\
& \leq\left(1-\alpha_{2}\right) \mathrm{E}_{2}\left[\left\|\left(1-\delta_{k+1}^{2}\right) A_{2} e_{k+1}^{2}\right\|_{2}^{2} \mid e_{k}\right]+\xi_{2}^{+} \\
& \leq\left(1-\alpha_{2}\right) \mathrm{E}_{2}\left[\left(1-\delta_{k+1}^{2}\right) \mid e_{k}\right] . \mathrm{E}_{2}\left[\left\|A_{2} e_{k+1}^{2}\right\|_{2}^{2} \mid e_{k}\right]+\xi_{2}^{+} \\
& \leq\left(1-\alpha_{2}\right) c_{2} \mathrm{E}_{2}\left[\left\|A_{2} e_{k+1}^{2}\right\|_{2}^{2} \mid e_{k}\right]+\xi_{2}^{+} \\
& \leq\left(1-\alpha_{2}\right) c_{2}\left\|A_{2}\right\|_{2}^{2} \mathrm{E}_{2}\left[\left\|e_{k+1}^{2}\right\|_{2}^{2} \mid e_{k}\right]+\xi_{2}^{+} \\
& \leq\left(1-\alpha_{2}\right) c_{2}\left(\left\|A_{2}\right\|_{2}^{2}\right)^{2}\left\|e_{k}^{2}\right\|_{2}^{2}+\left(1-\alpha_{2}\right) c_{2} n_{2}\left\|A_{2}\right\|_{2}^{2}+\xi_{2}^{+} \\
& \leq\left(1-\alpha_{2}\right) c_{2}\left(\left\|A_{2}\right\|_{2}^{2}\right)^{2} \sum_{i=1,2}\left\|e_{k}^{i}\right\|_{2}^{2}+\xi_{3}^{+}
\end{aligned}
$$

where, the second inequality insured through independence of $\delta_{k+1}^{2}$ and $e_{k+1}^{2}$. Moreover, $\xi_{2}^{+}=\xi^{+}+\alpha_{2} \varepsilon_{2}\left\|A_{2}\right\|_{2}^{2}$, and $\xi_{3}^{+}=\xi_{2}^{+}+\left(1-\alpha_{2}\right) c_{2} n_{2}\left\|A_{2}\right\|_{2}^{2}$. The drift in (11) then yields

$$
\begin{aligned}
& \Delta^{2} V\left(e_{k}\right)=\mathrm{E}\left[V\left(e_{k+2}\right) \mid e_{k}\right]-V\left(e_{k}\right) \\
& \leq \quad\left(1-\alpha_{2}\right) c_{2}\left(\left\|A_{2}\right\|_{2}^{2}\right)^{2} \sum_{i=1,2}\left\|e_{k}^{i}\right\|_{2}^{2}+\xi_{3}^{+}-\sum_{i=1,2}\left\|e_{k}^{i}\right\|_{2}^{2} \\
& =\left[\left(1-\alpha_{2}\right) c_{2}\left(\left\|A_{2}\right\|_{2}^{2}\right)^{2}-1\right] \sum_{i=1,2}\left\|e_{k}^{i}\right\|_{2}^{2}+\xi_{3}^{+} \leq-f(e)
\end{aligned}
$$

We define $f(e)=\bar{\varepsilon}_{f} \sum_{i=1,2}\left\|e_{k}^{i}\right\|_{2}^{2}-\xi_{3}^{+}, \bar{\varepsilon}_{f}>0$, knowing $\xi_{3}^{+}$is bounded. Then, choosing $\bar{\varepsilon}_{f}$ such that $\left[\left(1-\alpha_{2}\right) c_{2}\left(\left\|A_{2}\right\|_{2}^{2}\right)^{2}-1\right] \leq-\bar{\varepsilon}_{f}$ implies $\Delta^{2} V\left(e_{k}\right) \leq$ $-f(e)$. We can find an appropriate $\bar{\varepsilon}_{f}$ and a compact set $\mathcal{D}$ such that $f \geq 1$, and

$$
\left(1-\alpha_{2}\right) c_{2}<\frac{1}{\left(\left\|A_{2}\right\|_{2}^{2}\right)^{2}} \quad \text { for all } \quad e \notin \mathcal{D}
$$

to satisfy (14). It guarantees by (15) that

$$
\mathrm{E}\left[\left(1-\delta_{k+1}^{2}\right) \mid e_{k}\right] \leq \frac{1}{\left(1-\alpha_{2}\right)\left(\left\|A_{2}\right\|_{2}^{2}\right)^{2}}
$$

and $f$-ergodicity of $e_{k}$ is then followed. Second moment boundedness of the process $e_{k}$ is accordingly followed by 
finiteness of the stationary distribution $\pi(f)$, as in Def. 1 . Moreover, it is clear from (14) that if $e \in \mathcal{D}$, then $\Delta^{2} V<\infty$ and the stochastic stability of $e_{k}$ is evident.

\section{N-Subsystem Network}

In this section the stability of a NCS with arbitrary finite number of heterogeneous subsystems will be presented. We first state the following essential lemma.

Lemma 2: Suppose that $\delta_{k}^{i}=1$ for some $k$ and $i$. If there exists a subsystem $j \neq i$ such that $\left\|e_{k+N}^{j}\right\|_{2}^{2}>M_{j}$, then the probability that $\delta_{k+N}^{i}=1$ is upper bounded by

$$
\mathrm{P}\left[\delta_{k+N}^{i}=1 \mid\left\|e_{k+N}^{j}\right\|_{2}^{2}>M_{j}, \delta_{k}^{i}=1\right] \leq \frac{\sum_{k=0}^{N-1} n_{j}\left\|A_{j}^{k}\right\|_{2}^{2}}{M_{j}}
$$

Proof: Taking the definition of the PEB policy in (5) and employing iterated expectation law

$$
\begin{aligned}
& \mathrm{P}\left[\delta_{k+N}^{i}=1 \mid\left\|e_{k+N}^{j}\right\|_{2}^{2}>M_{j}, \delta_{k}^{i}=1\right] \\
& =\mathrm{E}\left[\mathrm{P}\left[\delta_{k+N}^{i}=1 \mid e_{k}\right] \mid\left\|e_{k+N}^{j}\right\|_{2}^{2}>M_{j}, \delta_{k}^{i}=1\right] \\
& =\mathrm{E}\left[\frac{\left\|e_{k+N}^{i}\right\|_{2}^{2}}{\sum_{j=1}^{N}\left\|e_{k+N}^{j}\right\|_{2}^{2}} \mid\left\|e_{k+N}^{j}\right\|_{2}^{2}>M_{j}, \delta_{k}^{i}=1\right] \\
& \leq \mathrm{E}\left[\frac{\left\|e_{k+N}^{i}\right\|_{2}^{2}}{M_{j}} \mid e_{k}, \delta_{k}^{i}=1\right] \leq \frac{\sum_{k=0}^{N-1} n_{j}\left\|A_{j}^{k}\right\|_{2}^{2}}{M_{j}}
\end{aligned}
$$

Above lemma implies that the probability of two subsequent transmissions of the same subsystem within a fixed interval can be made arbitrarily small by choosing $M_{j}$ accordingly.

Theorem 3: Let a NCS consists of $N$ arbitrary stochastic LTI control loops sharing a communication channel subject to the constraint in (6). Suppose that the stabilizing controller $\gamma^{i}$ is given as (2). Then, the Markov chain $e_{k}$ in (7), is $f$ ergodic and has finite second moment, if the channel access is scheduled according to the PEB scheme introduced in (5).

Proof: We evaluate the stability over $N$ time steps. As in Def. 2, introduce the quadratic function $V\left(e_{k}\right)=$ $\sum_{i=1}^{N}\left\|e_{k}^{i}\right\|_{2}^{2}$, and the $N$ th-order drift operator $\Delta^{N} V\left(e_{k}\right)=$ $\mathrm{E}\left[V\left(e_{k+N}\right) \mid e_{k}\right]-V\left(e_{k}\right)$. The error evolves then according to the following finite memory Markov chain

$$
\begin{aligned}
e_{k+N}^{i} & =\prod_{j=0}^{N-1}\left(1-\delta_{k+j}^{i}\right) A_{i}^{N} e_{k}^{i}+\prod_{j=1}^{N-1}\left(1-\delta_{k+j}^{i}\right) A_{i}^{N-1} w_{k}^{i} \\
& +\ldots+\left(1-\delta_{k+N-1}^{i}\right) A_{i} w_{k+N-2}^{i}+w_{k+N-1}^{i}
\end{aligned}
$$

Then, we have for $N$ time steps later, $\mathrm{E}\left[V\left(e_{k+N}\right) \mid e_{k}\right]$ as

$$
\begin{aligned}
& \mathrm{E}\left[V\left(e_{k+N}\right) \mid e_{k}\right]=\sum_{i=1}^{N} \mathrm{E}\left\|\prod_{j=0}^{N-1}\left(1-\delta_{k+j}^{i}\right) A_{i}^{N} e_{k}^{i}\right\|_{2}^{2} \\
& +\quad \sum_{i=1}^{N} \mathrm{E}\left\|\prod_{j=1}^{N-1}\left(1-\delta_{k+j}^{i}\right) A_{i}^{N-1} w_{k}^{i}\right\|_{2}^{2}+\ldots \\
& +\sum_{i=1}^{N} \mathrm{E}\left\|\left(1-\delta_{k+N-1}^{i}\right) A_{i} w_{k+N-2}^{i}\right\|_{2}^{2}+\sum_{i=1}^{N} \mathrm{E}\left\|w_{k+N-1}^{i}\right\|_{2}^{2}
\end{aligned}
$$

To continue the proof, we divide all the possible situations into three complementary cases in terms of transmission ordering and size of error, with each of the cases occurring with a probability $\mathrm{P}_{\bar{c}_{l}} \in[0,1]$, and $\sum_{l=1}^{3} \mathrm{P}_{\bar{c}_{l}}=1$, during the $N$ time-step period. The cases are as follows:

$l=1$ Every subsystem $i \in[1, \ldots, N]$ transmits merely once during the $N$ time-step period.

$l=2$ There exists at least one $i \in[1, \ldots, N]$ s.t. $\delta_{k^{\prime}}^{i}=0$ for all $k^{\prime} \in[k, k+N-1]$, and $\left\|e_{k^{\prime}}^{i}\right\|_{2}^{2} \leq M_{i}$.

$l=3$ There exists a non-empty set of subsystems $m$ s.t. for all $i \in m, \delta_{k^{\prime}}^{i}=0$ for all $k^{\prime} \in[k, k+N-1]$, and $\left\|e_{k^{\prime}}^{i}\right\|_{2}^{2}>M_{i}$ for at least one time-step $k^{\prime} \in$ $[k, k+N-1]$.

Since, only one of the cases $l \in[1,3]$ occurs in $N$ time-step period, the drift can be rewritten for each of the cases as

$$
\Delta^{N} V\left(e_{k}, l\right)=\mathrm{E}\left[V\left(e_{k+N}\right) \mid e_{k}, l\right]-V\left(e_{k}, l\right)
$$

Therefore, showing that the drift is negative outside a compact set $\mathcal{D}$ for each of the aforementioned cases, guarantees the drift is always negative. For further derivations, we divide the set of subsystems into two complementary subsets. The set $s_{1}$ consists of the $\bar{m}$ subsystems which are granted the resource access at least once during the $N$ time-step network operation, and the set $s_{2}$ contains the $m=N-\bar{m}$ subsystems which have not transmitted at all. In the other words, the scheduler variable $\delta_{k+j}^{i \in s_{1}}=1$ at least for one $j$, and $\delta_{k+j}^{i \in s_{2}}=0$ for all $j$ where $j \in\{0,1, \ldots, N-1\}$. In terms of channel access, unlike the first case at which all the subsystems eventually transmit during the $N$ time-step period, in the second and third cases, not all the subsystems transmit, i.e. there is at least one subsystem $i$ s.t. $\delta_{k^{\prime}}^{i}=0$ for all $k^{\prime} \in[k, k+N-1]$. In terms of allowable error size though, the first case places no boundary on the size of the error norm, unlike the second and third cases.

The first case, $l=1$, ensures that every subsystem $i$, is assigned with a time-step $k+j$ with $j \in[0, \ldots, N-1]$, s.t. $\delta_{k+j}^{i}=1$. It implies that the first summation in (16), which is the only error-dependent term, is zero. The other noise-dependent terms in (16) are bounded for finite $N$. Therefore, the first component of the drift in (17), $\mathrm{E}\left[V\left(e_{k+N}\right) \mid e_{k}, l=1\right]$ is trivially bounded. Showing the drift is negative follows the same approach as in Theorem 2.

For the second case, we divide the drift as follows

$$
\begin{aligned}
\mathrm{E} & {\left[V\left(e_{k+N}\right) \mid e_{k}, l=2\right] } \\
& =\mathrm{E}\left[V\left(e_{k+N}\right) \mid e_{k}, i \in s_{1}\right]+\mathrm{E}\left[V\left(e_{k+N}\right) \mid e_{k}, i \in s_{2}\right] \\
& \leq \sum_{i \in s_{2}}\left[\mathrm{E}\left\|\left(1-\delta_{k+N-1}^{i}\right) A_{i} e_{k+N-1}^{i}\right\|_{2}^{2} \mid e_{k}\right]+\xi^{+}
\end{aligned}
$$

where, $\xi^{+}$stands for the sum of the bounded noise-dependent terms in (16). The first summation in (16) is zero for $i \in s_{1}$. Introduce the binary variable $d_{i} \in\{1,2\}$

$$
d_{i}= \begin{cases}1 & \left\|e_{k+N-1}^{i}\right\|_{2}^{2} \leq \varepsilon_{i}<M_{i} \\ 2 & \left\|e_{k+N-1}^{i}\right\|_{2}^{2}>\varepsilon_{i}\end{cases}
$$


with $\mathrm{P}\left(d_{i}=1 \mid e_{k}\right)=\alpha_{i}$ and $\mathrm{P}\left(d_{i}=2 \mid e_{k}\right)=1-\alpha_{i}$. Thus, the law of iterated expectation incurs

$$
\begin{aligned}
\mathrm{E} & {\left[\left\|\left(1-\delta_{k+N-1}^{i}\right) A_{i} e_{k+N-1}^{i}\right\|_{2}^{2} \mid e_{k}\right] } \\
& =\mathrm{E}\left[\mathrm{E}\left[\left\|\left(1-\delta_{k+N-1}^{i}\right) A_{i} e_{k+N-1}^{i}\right\|_{2}^{2} \mid e_{k}, d\right] \mid e_{k}\right] \\
& =\mathrm{P}\left(d_{i}=1 \mid e_{k}\right) \cdot \mathrm{E}_{1}\left[\left\|\left[1-\delta_{k+N-1}^{i}\right] A_{i} e_{k+N-1}^{i}\right\|_{2}^{2} \mid e_{k}\right] \\
& +\mathrm{P}\left(d_{i}=2 \mid e_{k}\right) \cdot \mathrm{E}_{2}\left[\left\|\left[1-\delta_{k+N-1}^{i}\right] A_{i} e_{k+N-1}^{i}\right\|_{2}^{2} \mid e_{k}\right] \\
& \leq \varepsilon_{i} \alpha_{i}\left\|A_{i}\right\|_{2}^{2}+\left(1-\alpha_{i}\right) \mathrm{E}_{2}\left[\left\|\left[1-\delta_{k+N-1}^{i}\right] A_{i} e_{k+N-1}^{i}\right\|_{2}^{2} \mid e_{k}\right]
\end{aligned}
$$

It readily follows from the policy definition in (5) that

$$
\mathrm{E}_{2}\left[1-\delta_{k+N-1}^{i} \mid e_{k}\right]=\mathrm{E}_{2}\left[\frac{\sum_{j \neq i}^{N}\left\|e_{k+N-1}^{j}\right\|_{2}^{2}}{\sum_{j=1}^{N}\left\|e_{k+N-1}^{j}\right\|_{2}^{2}} \mid e_{k}\right]
$$

Lemma 2 ensures that, with probability arbitrarily close to one, all the subsystems in $s_{1}$ have bounded errors, so

$\mathrm{E}_{2}\left[\frac{1}{\sum_{j=1}^{N}\left\|e_{k+N-1}^{j}\right\|_{2}^{2}} \mid e_{k}\right] \leq \mathrm{E}_{2}\left[\frac{1}{\sum_{j \in s_{2}}\left\|e_{k+N-1}^{j}\right\|_{2}^{2}} \mid e_{k}\right] \leq \frac{1}{\varepsilon_{i}}$

Existing a time-step $k^{\prime}$ s.t. $\left\|e_{k^{\prime}}^{i}\right\|_{2}^{2}<M_{i}$ and the discussions in Lemma 2, assure the boundedness of $\sum_{j=1}^{N \neq i} \mathrm{E}\left\|e_{k+N-1}^{j}\right\|_{2}^{2}$. Then, employing Lemma 1 provides an upper bound for (19)

$$
\begin{aligned}
& \mathrm{E}_{2}\left[1-\delta_{k+N-1}^{i} \mid e_{k}\right] \\
& \quad \leq \frac{\sum_{j \neq i}^{N} \mathrm{E}\left\|e_{k+N-1}^{j}\right\|_{2}^{2}}{\varepsilon_{i}}-\sum_{i_{(\text {Odd })}=1}^{\infty} \frac{(i+1) !\left(2 \sigma^{2}\right)^{\left(\frac{i+1}{2}\right)}}{2^{i+1}\left(\frac{i+1}{2}\right) E[b]^{i+1}}=c_{i}
\end{aligned}
$$

where, $\sigma^{2}$ is $\operatorname{Var} \sum_{j \neq i}^{N}\left[\left\|e_{k+N-1}^{j}\right\|_{2}^{2}-\mathrm{E}\left\|e_{k+N-1}^{j}\right\|_{2}^{2}\right]$, and $b=\sum_{i=1}^{N}\left\|e_{k+N-1}^{i}\right\|_{2}^{2}$. Summing up both bounds yields

$$
\begin{aligned}
\mathrm{E} & {\left[V\left(e_{k+N}\right) \mid e_{k}, l=2\right] } \\
& \leq\left(1-\alpha_{i}\right) \sum_{i \in s_{2}} \mathrm{E}_{2}\left[\left\|\left(1-\delta_{k+N-1}^{i}\right) A_{i} e_{k+N-1}^{i}\right\|_{2}^{2} \mid e_{k}\right]+\xi_{2}^{+} \\
& \leq\left(1-\alpha_{i}\right) \sum_{i \in s_{2}} c_{i}\left\|A_{i}\right\|_{2}^{2} \mathrm{E}_{2}\left[\left\|e_{k+N-1}^{i}\right\|_{2}^{2} \mid e_{k}\right]+\xi_{2}^{+} \\
& \leq\left(1-\alpha_{i}\right) \sum_{i \in s_{2}} c_{i}\left(\left\|A_{i}\right\|_{2}^{2}\right)^{N} V\left(e_{k}\right)+\xi_{3}^{+}
\end{aligned}
$$

where, $\xi_{2}^{+}=\xi^{+}+\sum_{i \in s_{2}} \alpha_{i} \varepsilon_{i}\left\|A_{i}\right\|_{2}^{2}$, and $\xi_{3}^{+}=\xi_{2}^{+}+$ $\mathrm{E}_{2}\left[\left\|A_{i}^{N-2} w_{k}^{i}+\ldots+A_{i} w_{k+N-3}^{i}+w_{k+N-2}^{i}\right\|_{2}^{2} \mid e_{k}\right]$, which is bounded for finite $N$. The $N$ th order drift then becomes

$$
\begin{aligned}
& \Delta^{N} V\left(e_{k}, l=2\right) \\
& \quad \leq\left[\left(1-\alpha_{i}\right) \sum_{i \in s_{2}} c_{i}\left(\left\|A_{i}\right\|_{2}^{2}\right)^{N}-1\right] \sum_{j=1}^{N}\left\|e_{k}^{j}\right\|_{2}^{2}+\xi_{3}^{+}
\end{aligned}
$$

Define $f(e)=\bar{\varepsilon}_{f} V\left(e_{k}\right)-\xi_{3}^{+}, \bar{\varepsilon}_{f}>0$. Then, choosing $\bar{\varepsilon}_{f}$ such that $\left[\left(1-\alpha_{i}\right) \sum_{i \in s_{2}} c_{i}\left(\left\|A_{i}\right\|_{2}^{2}\right)^{N}-1\right] \leq-\bar{\varepsilon}_{f}$ implies $\Delta^{N} V\left(e_{k}, l=2\right) \leq-f(e)$. We can find an appropriate $\bar{\varepsilon}_{f}$ and a compact set $\mathcal{D}$ such that $f \geq 1$, and

$$
\left(1-\alpha_{i}\right) \sum_{i \in s_{2}} c_{i}\left(\left\|A_{i}\right\|_{2}^{2}\right)^{N} \leq 1 \quad \text { for all } e \notin \mathcal{D}
$$

and $f$-ergodicity of $e_{k}$ is then followed according to Theorem 1. Second moment boundedness of the Markov chain $e_{k}$ is also shown by finite stationary distribution $\pi(f)$, in Def. 1 . Clearly, if $e \in \mathcal{D}$, then $\Delta^{N} V\left(e_{k}, l=2\right)<\infty$, since $\xi_{3}^{+}$is bounded.

The third case considers multiple subsystems with unbounded errors $\left\|e_{k^{\prime}}^{i}\right\|_{2}^{2}$ for some time-steps $k^{\prime}$. Considering (19), we may exclude the subsystems who transmit at least once, i.e. $j \in s_{1}$, because they have bounded errors. Hence, (19) can be rewritten as

$\mathrm{E}_{2}\left[1-\delta_{k+N-1}^{i} \mid e_{k}\right]=\mathrm{E}_{2}\left[\frac{\sum_{j \in s_{2}}^{j \neq i}\left\|e_{k+N-1}^{j}\right\|_{2}^{2}}{\sum_{j \in s_{2}}\left\|e_{k+N-1}^{j}\right\|_{2}^{2}} \mid e_{k}\right]+\epsilon<1$

where, $\epsilon \in(0,1)$ replaces the effect of excluded subsystems $j \in s_{1}$. Furthermore, $e_{k+N-1}^{i}$ is a linear combination of the independent zero-mean random variables $e_{0}^{i}, w_{0}^{i}, \ldots, w_{k-1}^{i}$, and it has a standard normal distribution with the bounded variance $A_{i}^{k+N-1} \sigma_{e_{0}^{i}}^{2}+\sum_{l=1}^{k+N-1} A_{i}^{k-l}$. Thus, $\left\|e_{k+N-1}^{i}\right\|_{2}$ has a central chi distribution. Since $e_{k+N-1}^{i}$, are independent for $i \in\{1, \ldots, N\}, \mathrm{E}\left[\sum_{i=1}^{N}\left\|e_{k+N-1}^{i}\right\|_{2}^{2} \mid e_{k}\right]=$ $\sum_{i=1}^{N} \mu_{i}$, and $\mathrm{E}\left[\left[\sum_{i=1}^{N}\left[\left\|e_{k+N-1}^{i}\right\|_{2}^{2}-\mu_{i}\right]\right]^{i+1} \mid e_{k}\right]=$ $\left(2 \sum_{i=1}^{N} \mu_{i}\right)^{\frac{i+1}{2}} i$ !!, with $\mu_{i}$ the second moment of the chi distributed random variable $\left\|e_{k+N-1}^{i}\right\|_{2}$, and '!!' represents the odd factorial. According to Lemma 1 , if $a \simeq b$

$$
\mathrm{E}[a] . \mathrm{E}\left[\frac{1}{a}\right]-\sum_{i_{\text {Odd }}=1}^{\infty} \frac{\mathrm{E}\left[(a-\mathrm{E}[a])^{i+1}\right]}{(\mathrm{E}[a])^{i+1}}<1
$$

Define $a=\sum_{j \in s_{2}}^{j \neq i}\left\|e_{k+N-1}^{j}\right\|_{2}^{2}$ and $b=\sum_{j \in s_{2}}\left\|e_{k+N-1}^{j}\right\|_{2}^{2}$, the bound can be rewritten as

$$
\begin{gathered}
\mathrm{E}_{2}\left[\frac{\sum_{j \in s_{2}}^{j \neq i}\left\|e_{k+N-1}^{j}\right\|_{2}^{2}}{\sum_{j \in s_{2}}\left\|e_{k+N-1}^{j}\right\|_{2}^{2}} \mid e_{k}\right] \leq \frac{\mathrm{E}\left[\sum_{j \in s_{2}}\left\|e_{k+N-1}^{j}\right\|_{2}^{2}\right]}{\varepsilon_{i}} \\
-\sum_{i_{\text {Odd }}=1}^{\infty} \frac{2^{\frac{i+1}{2}}\left[\sum_{i \in s_{2}} \mu_{i}\right]^{\frac{i+1}{2}} i ! !}{\left[\sum_{i \in s_{2}} \mu_{i}\right]^{i+1}}<1
\end{gathered}
$$

The boundedness of $\mathrm{E}\left[\sum_{j \in s_{2}}\left\|e_{k+N-1}^{j}\right\|_{2}^{2} \mid e_{k}\right]$ is immediately followed by the boundedness of the last summation. Finally, we can find the finite upper bound for the drift as

$$
\begin{aligned}
\mathrm{E} & {\left[V\left(e_{k+N}\right) \mid e_{k}, l=3\right] } \\
& \leq\left(1-\alpha_{i}\right) \sum_{i \in s_{2}}\left\|A_{i}\right\|_{2}^{2} \mathrm{E}_{2}\left[\left\|e_{k+N-1}^{i}\right\|_{2}^{2} \mid e_{k}\right]+\xi_{2}^{+} \\
& \leq\left(1-\alpha_{i}\right)\left\|A_{i}\right\|_{2}^{2 m} \sum_{i \in s_{2}} \mathrm{E}_{2}\left[\left\|e_{k+N-1}^{i}\right\|_{2}^{2} \mid e_{k}\right]+\xi_{2}^{+} \\
& \leq\left(1-\alpha_{i}\right)\left\|A_{i}\right\|_{2}^{2 m N} \sum_{i \in s_{2}} V\left(e_{k}\right)+\xi_{3}^{+}
\end{aligned}
$$

where, $\xi_{2}^{+}$and $\xi_{3}^{+}$are already defined, and $m$ is the number of subsystems in the set $s_{2}$. The proof then follows as for the case $l=2$. Thus, the drift is negative for each of the three cases. Moreover, it is straightforward to see that the drift is bounded if the Markov chain remains inside a compact set $\mathcal{D}$ if $N$ is finite, according to the last inequality and the proof is then complete according to the Aperiodic Ergodic Theorem. 


\section{NUMERICAL RESULTS}

Suppose the system comprises of two classes of subsystems - a stable and an unstable process - with system parameters $A_{1}=1.25, B_{1}=1$ and $A_{2}=0.75, B_{2}=1$, respectively. In both classes, the state starts with $x_{0}^{1}=x_{0}^{2}=$ 0 and the random disturbance is given by $w_{k}^{i} \sim \mathcal{N}(0,1)$. We consider $N$ subsystems with the two subsystem types occurring at a constant ratio. To stabilize the subsystems, we assume a deadbeat control law with $L_{i}=A_{i}$ for $i \in\{1,2\}$ and a model-based observer given by (3).

Figure 2 compares the proposed PEB protocol with other scheduling protocols for different $N \in\{2,4,6,8,10\}$ in terms of the mean squared of the estimation error $e_{k}^{i}$ induced by the network. It should be noted that for $N>2$, we have more unstable systems than the available transmission slots, which is one. The averages are calculated by their empirical means through Monte Carlo simulations over a horizon of 100000. The lower bound is determined by relaxing the initial problem to have no resource constraint, but instead restrains the total average transmission rate per time step to be 1 . The resulting scheduler can be calculated through a bilevel approach which is discussed in [12] and results in an event-triggered scheduling strategy. The round robin protocol is a time-triggered access scheme, where subsystems update their controllers periodically with a sampling period of $N$. The carrier sense multi access protocol considered operates in the same fashion as the PEB protocol without prioritizing subsystems, i.e. the probability of updating the controller is $\frac{1}{N}$ for each subsystem at each time. With increasing number of subsystems sharing the resource, the performance efficiency of the PEB scheduler becomes more evident with the other protocols. As it can be seen from Figure 3, only for $N=2$, the CSMA protocol results in an acceptable performance. For $N \geq 6$, the variance of $e_{k}$ takes values of magnitude $10^{15}$ which suggests an unbounded second moment. This is in accordance with theorem 2 in [12], where the stability condition is violated for $N \geq 6$ for the considered system parameters.

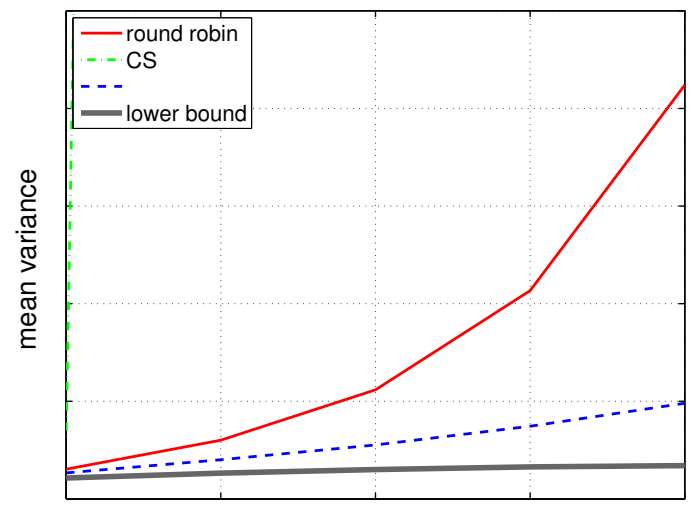

number of subsystems $N$

Fig. 2. Comparison of the mean steady-state variance of $e_{k}^{i}$ for various protocols and number of subsystems $N \in\{2,4, \ldots, 10\}$.

\section{CONCLUSIONS AND OUTLOOK}

In this paper, we propose a new stochastic scheduling scheme, Prioritized Error-Based (PEB), with dynamically assigned priorities for NCS comprised of a finite number of linear systems coupled through a common scarce communication resource, with no physical interconnection. Provided with stabilizing feedback controllers, we show the stability of the overall networked system under the employment of the PEB policy for the allocation of the resource using the drift criteria. In presence of stochastic disturbances, the stochastic stability is shown in terms of Markov chain ergodicity and second-order moment boundedness. Numerical simulations demonstrate the stability alongside a substantial performance improvement in comparison with the other randomized protocols, especially when the number of subsystems increases.

\section{REFERENCES}

[1] G. C. Walsh, H. Ye, and L. G. Bushnell, "Stability analysis of networked control systems," Control Systems Technology, IEEE Transactions on, vol. 10, no. 3, pp. 438-446, 2002.

[2] D. Nesic and A. Teel, "Input-output stability properties of networked control systems," Automatic Control, IEEE Transactions on, vol. 49, no. 10, pp. 1650-1667, 2004.

[3] W. H. Heemels, A. R. Teel, N. van de Wouw, and D. Nesic, "Networked control systems with communication constraints: Tradeoffs between transmission intervals, delays and performance," Automatic Control, IEEE Transactions on, vol. 55, no. 8, pp. 1781-1796, 2010.

[4] M. Tabbara and D. Nesic, "Input-output stability of networked control systems with stochastic protocols and channels," Automatic Control, IEEE Transactions on, vol. 53, no. 5, pp. 1160-1175, 2008.

[5] M. Donkers, W. Heemels, D. Bernardini, A. Bemporad, and V. Shneer, "Stability analysis of stochastic networked control systems," Automatica, vol. 48, no. 5, pp. 917-925, 2012.

[6] D. J. Antunes, J. P. Hespanha, and C. J. Silvestre, "Volterra integral approach to impulsive renewal systems: Application to networked control," Automatic Control, IEEE Transactions on, vol. 57, no. 3, pp. 607-619, 2012.

[7] Y. Xu and J. P. Hespanha, "Communication logic design and analysis for networked control systems," Current trends in nonlinear systems and control, pp. 495-514, 2006.

[8] S. Meyn and R. Tweedie, Markov chains and stochastic stability. Springer London, 1996.

[9] P. Tabuada, "Event-triggered real-time scheduling of stabilizing control tasks," Automatic Control, IEEE Transactions on, vol. 52, no. 9, pp. $1680-1685,2007$.

[10] J. Lunze and D. Lehmann, "A state-feedback approach to event-based control," Automatica, vol. 46, no. 1, pp. 211-215, 2010.

[11] A. Molin and S. Hirche, "On the optimality of certainty equivalence for event-triggered control systems," Automatic Control, IEEE Transactions on, vol. 58, no. 2, pp. $470-474,2013$.

[12] A. Molin and S. Hirche, "A bi-level approach for the design of eventtriggered control systems over a shared network," Discrete Event Dynamic Systems, 2013.

[13] X. Wang and M. D. Lemmon, "Event-triggering in distributed networked control systems," Automatic Control, IEEE Transactions on, vol. 56 , no. 3, pp. 586-601, 2011.

[14] M. Mamduhi, A. Molin, and S. Hirche, "On the stability of prioritized error-based scheduling for resource-constrained networked control systems," in 4th IFAC Workshop on Distributed Estimation and Control in Networked Systems (NecSys 13), To be presented, 2013.

[15] S. H. Rice, "A stochastic version of the price equation reveals the interplay of deterministic and stochastic processes in evolution," $B M C$ Evolutionary Biology, vol. 8, no. 1, p. 262, 2008.

[16] S. P. Meyn and R. Tweedie, "State-dependent criteria for convergence of markov chains," The Annals of Applied Probability, pp. 149-168, 1994.

[17] R. Lew, "Bounds on negative moments," SIAM Journal on Applied Mathematics, vol. 30, no. 4, pp. 728-731, 1976. 